\title{
Customer Centricity in Medical Affairs Needs Human-centric Artificial Intelligence
}

\author{
Alexander Bedenkov ${ }^{1}$ - Carmen Moreno ${ }^{2} \cdot$ Lyra Agustin $^{1} \cdot$ Nipun Jain $^{3}\left(\mathbb{D} \cdot\right.$ Amy Newman $^{4} \cdot$ Lana Feng $^{5}$. \\ Greg Kostello ${ }^{5}$
}

Accepted: 27 December 2020 / Published online: 19 January 2021

(c) The Author(s) 2021

\begin{abstract}
The evolution of healthcare, together with the changing behaviour of healthcare professionals, means that medical affairs functions of pharmaceutical organisations are constantly reinventing themselves. The emergence of digital ways of working, expedited by the COVID-19 pandemic, means that pharmaceutical-healthcare relationships are evolving to operate in an increasingly virtual world. The value of the pharmaceutical medical affairs function is dependent on understanding customers' needs and providing the right knowledge at the right time to physicians. This requires a human-centric artificial intelligence (AI) approach for medical affairs, which allows the function to query internal and external data sets in a conversational format and receive timely, accurate and concise intelligence on their customers.
\end{abstract}

\section{Key Points}

The medical affairs function in the pharmaceutical industry plays a role in enhancing knowledge of different stakeholders, often acting as scientific and healthcare partners to healthcare professionals (HCPs).

Medical affairs generate much data, but are not effectively able to use these data to generate insights on the identity of stakeholders or their needs.

Human-centric AI can be effective in solving this problem by starting with questions medical affairs teams are asking, and working backwards via machine learning (ML) powered natural language processing (NLP) engines to find answers from multiple data sets.

Nipun Jain

Nipun.Jain@astrazeneca.com

1 AstraZeneca Medical Affairs, International Region, Luton, UK

2 AstraZeneca Medical Affairs, International Region, Madrid, Spain

3 AstraZeneca Medical IT, International Region, Bangalore, India

4 AstraZeneca Global Medical Affairs, BioPharmaceutical Business, Gaithersburg, MD, USA

5 HUMA.AI, San Francisco, CA, USA

\section{Speed of Science}

The increasing need to deliver better healthcare services to patients brings a multitude of new players within the healthcare sector, driving the speed of innovation at different levels of the healthcare delivery system. The opening of strategic partnerships within the pharmaceutical industry and the new players, such as software developers, academe, digital healthcare start-ups to achieve novel solutions for patients, requires evolution in the old ways of the pharmaceutical industry, and in this instance, medical affairs, used to deliver its objectives. Digitalisation offers an innovative solution that will facilitate the transformation of medical affairs to an even more strategic function within the pharmaceutical organisation.

\subsection{A Brief History of the Medical Affairs Function}

The medical affairs function, in its current form, originated in 1967 at the Upjohn Company through the introduction of a field-based medical team. A small group of technically oriented sales representatives was formed with the goal of improving the image of the company with researchers, key opinion leaders (KOLs), also known as key external experts (KEEs) and investigators. These 'Medical Science Liaisons' (MSLs) utilised face-to-face peer interactions to better understand what their customers needed and to leverage 
insights from these interactions to shape products developed by the Upjohn Company into ongoing research activities [1].

In the same year, the Upjohn Company trademarked the 'MSL' term as 'Educational services-namely, initiation of drug studies in laboratory and clinical settings and development of workshop symposia and seminars for physicians, medical societies, specialty organisations, academicians, in concert, concerned with drug-related medical topics" [2].

Over the subsequent few decades, medical affairs teams were mostly operational, reporting into, and primarily supporting, marketing activities. While the healthcare authorities encouraged the formation of independent medical affairs departments, there was not a rigid set of requirements that dictated how such departments should look or operate. As such, the industry has developed a wide variety of models over the past 20 years, all seeking to address the intensified public and regulatory scrutiny applied to the pharmaceutical and medical device industries [4]. Operating guidelines for industry to ensure interactions with HCPs are independent, fair balanced, medically focused, unbiased and separate from commercial interest, including responding to unsolicited offlabel medical information requests, have been set out by the US Food and Drug Administration (FDA) [5, 6].

Starting in the mid-2000s, driven by the voluntary Code on Interactions with Healthcare Professionals by the Pharmaceutical Research and Manufacturers of America (PhRMA), most companies began establishing a 'firewall' between the marketing and medical affairs functions. As a result, the scope of activities within medical affairs expanded to include several areas beyond the provision of scientific and medical information [7] (see Fig. 1).
With the emergence of additional stakeholders, the healthcare environment will involve more complexities. From 2010, the function of health economics and outcomes research (HEOR) was increasingly included within the medical affairs function, followed by the expansion of the traditional KEEs concept, to include patient advocates, regulatory authorities and payors. Medical affairs would play a role in enhancing the knowledge of different stakeholders, thus helping them to gain a greater visibility and voice in decision making.

Over the last few years, ground-breaking digital technologies, the convergence of escalating priorities and unmet needs in wellness, with overlapping medical and consumer categories in healthcare have transformed the landscape and redefined the expectations and scope of the medical affairs function. Big data, artificial intelligence (AI), real-world evidence and the establishment of partnerships in fully integrated patient-centred ecosystems have begun to play a role in medical affairs strategy.

\subsection{Reimagining the Field Medical Function}

The pharmaceutical industry is uniquely placed to leverage scientific expertise, tools and capabilities to shape medical practice, which elevates the healthcare environment and accelerates innovation. Forward-thinking pharmaceutical companies are evolving the role of medical affairs and are positioning the function to create a competitive advantage [8].

There has been a gradual evolution in the relative field medical roles. The needs of HCPs, and the ways in which they want the pharmaceutical industry to relate to them,

\section{The Evolution of Medical Affairs}

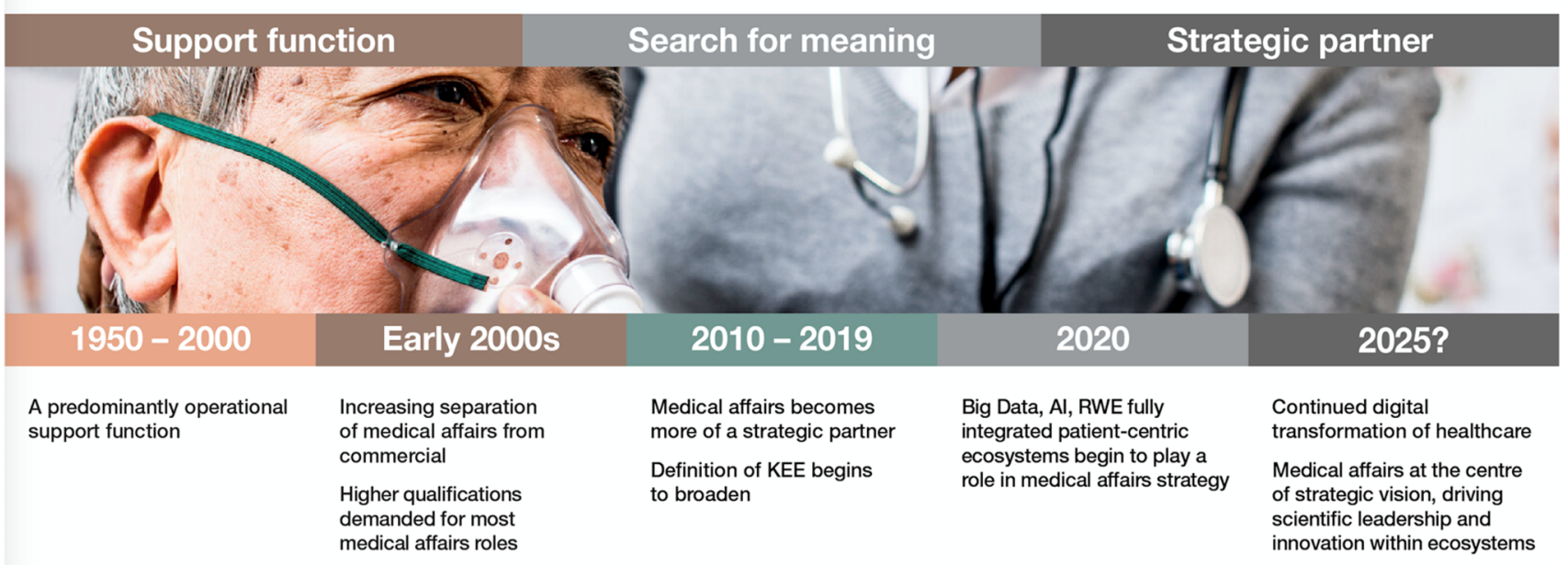

Fig. 1 A brief history of the medical affairs function through time [3]. AI artificial intelligence, $K E E$ key external expert, $R W E$ real-world evidence 
are changing. HCPs want a scientific and healthcare partner, as opposed to the traditional 'customer and seller' relationship.

As the pace of change has accelerated, now is the right time to evaluate how field activities should look postCOVID-19 pandemic - we want to define the future, not just follow it. Field medical roles create an invaluable bridge between HCPs and pharmaceutical companies-a vital connection that is based on scientific engagement, partnership and education.

By reimagining the ways of working within the medical affairs function, we prepare it to be future-proof through an intensified focus on new skills that will drive innovation and creativity in response to fast-paced healthcare changes. The introduction of new field medical roles, such as 'Medical Educators' and 'Clinical Science Liaisons', can maximise pre- and post-launch scientific engagement, partnership and education. The way to unlock the potential of this new field medical team is to build a stronger primary care ecosystem that fosters prevention, early detection and disease management. Addressing the post-COVID-19 pandemic landscape by expanding field medical reach and introducing new channels and services to maximise the value provided to patients, HCPs and pharmaceutical industry.

\subsection{The Digital Acceleration of the Medical Affairs Team}

The pharmaceutical industry plays a critical role in the digital revolution of health care [9]. Opportunities for digital expansion have for some time been a clear priority, even before the COVID-19 pandemic; albeit more apparent when all day-to-day interactions moved online. In April 2020, FirstWord Pharma published a poll of physicians' engagement with pharmaceutical representatives, which suggests they prioritise their engagements with the medical affairs team. However, it also highlights that the pharmaceutical industry will struggle to engage HCPs outside of traditional face-to-face meetings [10].

This led to opportunities to innovate and work outside of medical affairs comfort zone and, over the course of several months, there was a dramatic increase in digital initiatives delivered and led by the medical affairs function. Not only is the function evolving in the way it interacts with customers, but it is also creating other channels for digital engagement, expanding the traditional customers and defining new digital stakeholders, collaborating with partners who have digital capabilities, in turn transforming the way information and insights are collected and analysed.

\section{Technology as a Catalyst for Transforming Medical Affairs}

Healthcare is a big industry, accounting for more than $10 \%$ of the gross domestic product (GDP) of most developed countries. Worldwide, healthcare statistics show that healthcare is one of the fastest growing industries in the world, already accounting for close to $10 \%$ of GDP on average [11]. The global healthcare industry was worth $\$ 8.45$ trillion in 2018 , and spending is projected to reach over $\$ 10$ trillion by 2022 [12].

\subsection{Artificial Intelligence in Healthcare and Pharmaceutical Industry}

The fast growth is partly driven by the digitalisation of healthcare, triggered in part by worldwide migration to electronic health records. In recent years, the adoption of AI (the ability of software to mimic human behaviour) and Machine Learning (ML; synthesising a large volume of data to understand patterns and creating models to predictions about similar data) technologies have further accelerated healthcare digitalisation. MIT Technology Review Insights in partnership with GE Healthcare conducted a survey in October 2019 [13]. They interviewed 908 professionals from healthcare institutions, including medical, business and administrative roles. Survey respondents were from the USA (70 \%) and the UK (30\%). Results showed that 7 out of 10 healthcare institutions surveyed have adopted, or are considering adopting, AI. Of those that have adopted AI, more than $82 \%$ reported that their deployments have already resulted in operational or workflow improvements.

$\mathrm{AI}$ is increasingly being adopted by the pharmaceutical industry (see Fig. 2). A meta-analysis conducted by Schuhmacher et al., looked at 21 pharmaceutical companies between 2014 and 2019. They found that the industry is in an "early mature" phase of using AI in research and development [14].

\section{Human-Centric Al for Medical Affairs}

The increasing need to provide better healthcare solutions to patients is just one of the many reasons that is driving the digital transformation of healthcare and pharmaceutical companies. This is being made possible through the continuous collaborative efforts of academe, software companies, and digital health companies, including start-up tech companies. Innovative AI solutions are playing an increasing role in digital transformation of healthcare. The goal of those who create AI models is to provide a solution that is as good 
Fig. 2 Several examples of using artificial intelligence in the pharmaceutical industry $[15$, 16]. $A I$ artificial intelligence

\section{Examples of Artificial Intelligence use in the pharmaceutical industry}

a) Mobile platform to improve health outcomes, the ability to recommend patients by means of real time data collection and thus improve patient outcomes.

b) Personalised medicine, the ability to evaluate big database of patient to recognise cure options using a cloud-based system.

c) Innovation, new start-up companies are combining AI in healthcare to nourish the innovation requirements of pharmaceutical companies.

d) Drug discovery, pharma companies in conjunction with software companies are trying to implement the most cutting-edge technologies in the costly and extensive process of drug discovery, such as the IBM Watson and Pfizer collaboration and Novartis use of machine learning algorithms to predict which untested compound might be worth exploring in more details.

e) Improvement of drug adherence, such as the collaboration between Abbvie and AiCure to enhance trial vigilance and improve drug adherence. The use of facial and image recognition algorithm to monitor adherence, patients take a video of themselves swallowing a pill using their smartphones, the AI-powered platform confirms that indeed the correct person swallowed the pill.

f) Pharmacovigilance, such as the collaboration between Bayer and Genpact to improve pharmacovigilance. In this collaboration, the use of Genpact's pharmacovigilance AI made it possible to monitor drug adherence and detect potential side effects much earlier. as, or even better than, the performance that a human being would produce under the same circumstances. While AI can be particularly good at analysing large amounts of data, it is just an algorithm and is not a substitute for judgement and understanding, which we believe can only come from humans.

\subsection{Understanding Human-centric Al}

Many AI implementations that are successful today are examples of narrow AI, which tries to mimic human behaviour for a particular, or narrow, task. AI can be trained to perform a task and then applied in combination with different AI technologies, based on the type of problem you want to solve. For instance, Alexa, the virtual assistant AI technology developed by Amazon, is good at recognising and responding to voice commands; but has no concept of understanding an image. Other types of narrow AI are trained to recognise a particular type of disease in a medical image, but do not respond directly to human communication. Different narrow AIs can be combined to solve more complex problems. For example, when we ask Google if it is going to rain today, one AI is used to understand what we are asking and another more complex AI is used to determine the answer.

Machine Learning (ML) is the process of analysing large volumes of data to understand patterns and make predictions about similar data to create other ML models. Before the development of $\mathrm{ML}$, programmers were required to write all the rules to make predictions based on all known situations. This was a nearly impossible task, rife with error and limitations, unless the data were quite simple and the outcomes consistent. It was also impossible to find new patterns without human intervention. With ML, algorithmic training discovers patterns in the data to make predictions. The more data you input to the system, the more likely it is that an ML model will discover patterns and correctly make accurate predictions.

Moreover, as data change the models can be retrained to account for these adjustments, resulting in 'evergreen' ML learning. This allows systems to see new patterns, such as the trends in patient populations during the COVID-19 pandemic. The ML systems do not have to be programmed to extract those insights; the ML models discover the new patterns.

Natural language processing (NLP) combines AI and ML to mimic and extract information and make predictions based on large bodies of unstructured textual data-this is critical in healthcare, where $80 \%$ of the data are unstructured [17]. NLP systems make predictions about what a user intended by a phrase. For instance, when you use Alexa or speak to Google, NLP is transforming sound wave patterns into sentences and then converting those sentences into commands or queries understood by other systems. NLP can translate what you intended and then another system deciphers these commands. It is important therefore that you can use phrases that have the same meaning or 'intent', and the system understands without you having to express the command in a specific way.

Furthermore, NLP can be used to analyse text to pull out patterns of meaning. This goes beyond a simple keyword search to find the intent of a command; for example, to find patterns in protocols and perform comparisons, or to find 
insights from thousands of in-person notes taken by physicians. While these types of tasks are particularly challenging for humans, they are good at using this collated information to determine relevance and develop courses of action. Further advances in NLP include question and answer systems, where you can pose a question of the data and NLP finds the answer.

Human-centric AI explains how the AI develops answers by providing both the source of the data and the process for its analysis and prediction. This mimics human behaviour and expectations. We should all be sceptical of any algorithmically derived answer, just as we are of human-derived answers. One approach is to mimic human expertise in how to solve a problem, by essentially crafting similar approaches but providing the ability to take the same approach many times faster and over much larger data sets. At each step of the algorithm, a subject matter expert is used to validate the results. AI can enhance the user experience by navigating those same human-like patterns across many different permutations based on human interest and report back patterns. Although possible, this would be arduous without humancentric AI

The other critical area is to start with data already enhanced by human intelligence, which is expressed in peerand expert-reviewed documents. Rather than starting with raw data, the system starts with the analysis already vetted by humans. If the analysis is valuable and correct, then correlating and finding patterns across many of those documents provides intelligence based on high-quality human knowledge.

ML models that evolve with human interaction are at the forefront of AI and have proven to be far more efficient, delivering superior results compared with stand-alone models. These models combine human intuition and desire to seek novelty. This synthesis of human interaction provides context biasing toward the information that humans deem important versus putting randomised weights on the data [18].

Generative Pre-trained Transformer 3 (GPT-3; OpenAI, San Francisco, CA, USA) [19] is considered the most advanced NLP prediction model and uses Wikipedia and information from books and other citable public data to train the system. In certain circumstances, it can accurately mimic human behaviour to create papers, prose and even computer programs. GPT-3 is still limited because, at this point, it cannot easily ingest new data, but it demonstrates that leveraging citable human knowledge to answer questions and to create derivative bodies of work is possible.

Examples of human-centric AI are evident from Google searches, where the outcome of the search is often determined by the user's interaction with Google.

\subsection{The Role of Al in Uplifting the Capabilities of Medical Affairs}

New technology, such as AI can increase the strategic importance of field medical teams within an organisation, which improves work experience, medical affairs value proposition and ultimately, patient outcomes. Currently, $\mathrm{AI}$ is being used within the pharmaceutical industry in pharmacovigilance, mobile platform to improve health outcomes, including treatment adherence and in drug discovery.

There are several areas where medical affairs can actively use AI, ML and algorithm capabilities to optimise impact on both internal and external activities. Medical affairs can gain insights from multiple internal databases, by using both structured and unstructured data captured from HCPs and KEEs in the field, to better align engagements within the medical strategy. AI can be used to accelerate and synthesise insights from real-world data. AI can also be used to optimise digital channels for medical content and to understand the patient experience. To build upon the capabilities of medical affairs, it is necessary to use AI tools in collaboration with right internal (IT/Data Science function) or external (vendor) partners for the development of analytics, insights and the use of various data assets.

The right partnerships provide the pathway to meeting the goal of enhanced capabilities within medical affairs. Highquality insights and analytics, and their application to decision making will be the mark of successful collaboration by the medical affairs function.

\subsection{Applying Human-Centric Al to Medical Affairs}

Establishing partnerships between medical affairs and tech start-ups could revolutionise the way healthcare data are analysed. Health tech start-ups offer uniquely intelligent analytical tools that aim to simplify the process of extracting insights from large volumes of data, using human 'natural language' queries. The advanced ML platform can quickly understand and respond to these queries in order to analyse both structured and unstructured data, potentially unlocking a wealth of strategic information that can be used to support future decisions within medical affairs.

\subsubsection{Using Al for Generating Insights for Medical Affairs}

Measuring field medical impact has often presented a challenge in the past, with a growing amount of data being gathered from multiple sources and tools. We now have an opportunity to measure that impact, optimising the use of our existing data through analytics and AI. We can process 
and analyse enormous volumes of information from many different sources, using human queries to transform the data into accessible insights. This means medical affairs teams can quickly query customer relationship management (CRM) and medical data sources (internal and external), triggering actionable insights and reports on field medical activity. To accomplish this, the technology always starts with the questions that humans deem critical. This means spending time with users within medical affairs to determine what questions they want to ask of their data, and the answers they need throughout the organisation. This humancentric approach means that the AI is purpose-focused to solve specific problems and can be validated using traditional means. In fact, by mimicking the method that humans take, validation can happen at every step in the process.

First, a team member can use the platform to answer questions directly back from the platform. There is nothing to learn; the platform is trained based on the medical affairs domain. Over time, with each human interaction, the system gets "smarter" and can answer a broader set of questions and do more in-depth analyses. Second, the entire organisation can garner insights from all the unstructured notes that the medical affairs team generate when they visit HCPs. Traditional ML methods only work with structured data (rows of numbers and fixed sets of text). But the critical value of MSLs is in the information they bring back from being in the field and engaging HCPs. Human-centric AI trained to understand the MSL notes means that the actionable insights can now be surfaced along with trends in that data. With COVID-19, understanding new trends that emerge becomes critical.
Lastly, human-centric AI means getting answers across public and private data silos. No more going from one data silo to another search for answers. The system seeks out the answer from multiple systems to get back answers, even combining answers from one system and another to deliver back a unified picture (see Fig. 3).

\subsubsection{Building our Capabilities to Meet Future Challenges}

Through this new approach, our medical affairs teams are uplifting their practical capabilities in areas that will be increasingly vital in the future, such as data analytics, AI and ML, that give medical affairs teams the skills they need to make the most of new technology and shape future ways of working.

Collaboration starts from ideation and continues through building a platform driven by a pilot group of domain experts. Since medical affairs teams rely on curating information from multiple data sources to effectively engage KEEs and HCPs, the end goal is to put all the data at their fingertips through AI and NLP. During the platform build, the team assessed how analysed data can inform business decisions, and explore future possibilities such as how to integrate different medical platforms and systems so that different data points can talk to each other, that will enable us to realise the full value of medical affairs by making them more effective scientific partners to HCPs.

Building user behaviour and adoption of this way of working may take some time, therefore, ensuring that the technology improves day-to-day activities is key; involving end users in co-creation of the platform and ensuring user feedback is heard.

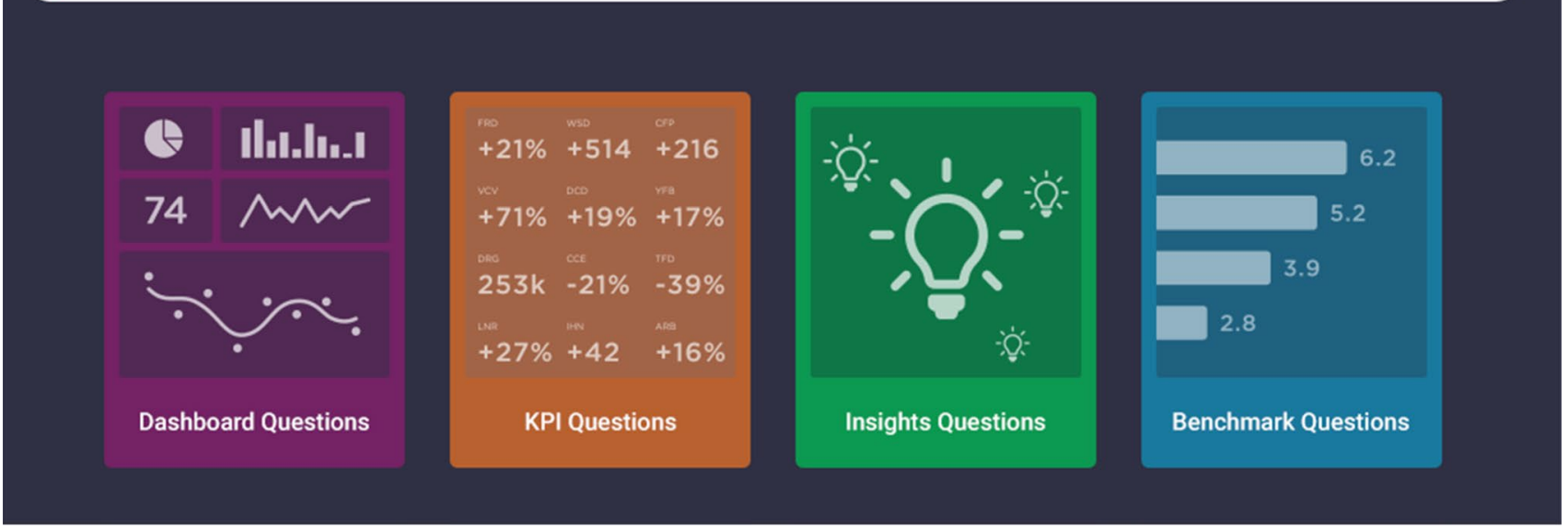

Fig. 3 Example of categories medical affairs team are interested in understanding using AI. a Summary dashboard of field medical activities, b KPI-related questions, $\mathbf{c}$ analysis of insights collected from the field and $\mathbf{d}$ benchmark data, comparing performance internally and externally. KPI key performance indicator 


\subsubsection{Making AI Work for the Medical Affairs Team}

Medical affairs functions, as with most pharmaceutical functions, manage an enormous amount of data [20]. The data are captured from field medical activities, such as interactions with physicians, and insights are generated. The data are then input into information technology (IT) systems, such as CRM, in standardised formats. Collecting and understanding data are not the same. Data analytics can be used to understand the data, which are generated in the form of dashboards populated with standard key performance indicators (KPIs).

The first element is data. The amount of data that an organisation has access to is not restricted to what is captured in the CRM system. Documents created by the medical affairs teams contain extensive information and insights, and often the information contained in documents, such as slide decks or spreadsheets, is more relevant to decision making than the structured information in an IT system. Looking externally, relevant information on HCP decision-making behaviour, preferences and insights can be gathered from social media [21]. Information on external experts can be gathered from the publications they author and the clinical trials they lead as investigators. Using a boundless approach in what is considered as data can broaden the scope of how decisions can be made.

Translating data into information and information into intelligence must also be considered. The more data sets that are used to drive decision making, the more important it becomes to understand the intelligence represented by the data. Converting data into intelligence has been the domain of data scientists, but now consuming structured and unstructured data sets to derive intelligence is possible via automated NLP- and ML- driven systems.

The second element is decision making. A time-tested traditional approach assumes that decision making is a standard process; in other words, everyone is using the same criteria to make decisions. This approach has been used in the creation of dashboards with a standard set of KPIs. However, this does not work well if non-standard, unique or new criteria need to be looked at requiring manual analysis of vast amounts of data, and the decision maker to act as data scientist. A better approach is to start with a KPI or a decisionmaker's question and work backwards towards an answer. This allows for a more natural decision-making process to happen, which means unique questions can be asked about specific situations or by different personalities. It enables an understanding of what data are needed to answer questions in this boundless way.

To allow domain experts in medical affairs who need data and insights to make real-time decisions, a new platform has been co-created by the authors, which follows a different approach from commercial data analytics platforms that mostly need a data science expertise to use. This ML enabled, question and answer-driven knowledge automation platform turns domain experts into their own data scientists, solving the last mile issue of applying data-driven insights to decision making. The self-service AI platform analyses both structured and unstructured data. More importantly, the platform is a light natural language understanding (NLU) meta-layer that sits on top of any existing data systems and connects the silos. ML and NLU are not only used at the front end, to understand the context of users' questions, map them to the correct data source(s) and analyse data to provide answers, it is also used at the back end to ingest unstructured data, be it free-text columns within a tabular dataset or stand-alone text documents.

One of our use cases is to connect public data sources to provide higher-level intelligence for domain experts. Data sources such as PubMed, ClinicalTrials.gov and OpenFDA offer a wealth of information to medical researchers. The challenge is that they are difficult to use for non-data scientists, requiring complex filtering and once filtered, manually going through search results one by one to extract insights. The platform, however, allows users to just ask questions and instantly find out thought leaders \& KEE activities from publications, clinical trials and even regulatory approval standpoint, therefore transcending data sources to obtain higher level intelligence that really matters to domain experts.

In essence, the traditional method of data-driven decision making has been modernised by removing the barriers that dictate what data sets are utilised and how data are converted into intelligence, and by decision makers asking questions rather than being presented with a list of predefined answers. Achieving this human-centric, AI-driven decision-making method required new-age thinking has been achieved by partnering with the right innovator.

\section{Conclusions}

In the era of big data, competitive pharmaceutical companies generate and analyse vast volumes of real-world data, and they must excel at communicating scientific evidence and help physicians navigate in a far more complex healthcare universe. To keep up with the landscape shift with emerging new technologies, leading pharmaceutical companies are reimagining and upgrading their medical affairs functions. Equipped with deep product knowledge and disease understanding, medical affairs teams are well positioned to generate and present high-quality scientific knowledge to the market and educate stakeholders about next-generation solutions. As physicians become more reliant on scientific sources of information, an experienced medical affairs team can link scientific and clinical results to patient outcomes, 
adding value at every stage of a medicine's development, transforming medical affairs to medical value.

Companies that leverage human-centric AI for medical affairs will build competitive advantage in a rapidly changing pharmaceutical landscape. The use of AI technology within medical affairs should be assessed against emerging technologies that automate adding human language interactions to any existing systems or databases. When transforming medical affairs, it is important to establish discussions with IT and data scientists to explore opportunities that might exist. For instance, some medical affairs groups are leveraging their data with a human-centric approach to better understand patient experiences and gain insights into the behaviours of thought leaders and HCPs. They align their work with thought leaders to match their medical strategy and use AI to improve upon the execution.

When working with AI/NLP, the tendency is to start with a data-centric approach that is focused on taxonomies, categorisation or themes, but with a human-centric approach, we start with a set of questions that need to be answeredwhether it is for field medical, medical information, clinical trials, or across all data areas, by tying them together into a shared comprehensive answer. This logical approach of asking the questions first allows us to reach past what we thought possible. It is not about how the data are structured or what categorised information is already known. AI/NLP can be a layer that sits external to existing systems, enhancing current applications without replacing them, avoiding costly system integrations. This layer serves as the connective tissue between silos with the ability to simply ask questions and receive answers within seconds, allowing end users to automatically access data, gain insights and take actions using everyday language. The focus is on the true end user 'self-serve' (ask and receive) and connecting data silos.

For any unknown questions asked of AI tools, they typically respond with "I don't understand" or "I don't know that", but capabilities exist that can give users direct visibility to the unanswered questions and provide feedback as the AI response is being resolved (trained). As the human factor is applied against known business rules the tool learns and adapts; this allows users to gain deeper insights and make better predictions with unknowns or 'golden nuggets' rising to the surface. Human-centric AI allows for the ability to obtain all relevant data, analyse them quickly, surface actionable insights and drive them back into operational systems to affect events as they are still unfolding. The advantage is to make fast and better decisions and quickly act on insights gained from large amounts of medical data.

Ultimately, medical affairs' most common use of $\mathrm{AI}$ is to synthesise real-world data insights and to align with KEE engagements. HCPs and thought leaders are key external stakeholders for data insights aligning with a digital strategy.
Creating a more formalised digital AI strategy within medical affairs can also extend to patients and patient advocacy groups looking for a way to create tools that allow for the education of patients and to deliver disease state education more readily. To boost the presence and effectiveness of medical affairs digital programs, first develop a clear digital strategy, execute on this digital strategy and adequately resource digital efforts.

\section{Declarations}

Funding No funding has been received for this manuscript. The open access fee was paid by AstraZeneca.

Conflict of interest All authors are employees of AstraZeneca or HUMA.AI. The opinions expressed in this article are those of the authors and not necessarily those of AstraZeneca or HUMA.AI.

Availability of data and material Not applicable.

Ethics approval Not applicable.

Consent to participate Not applicable.

Consent to publish Not applicable.

Code availability Not applicable.

Author contributions All authors were involved in writing the review, and read and approved the final version of the submitted paper.

Open Access This article is licensed under a Creative Commons Attribution-NonCommercial 4.0 International License, which permits any non-commercial use, sharing, adaptation, distribution and reproduction in any medium or format, as long as you give appropriate credit to the original author(s) and the source, provide a link to the Creative Commons licence, and indicate if changes were made. The images or other third party material in this article are included in the article's Creative Commons licence, unless indicated otherwise in a credit line to the material. If material is not included in the article's Creative Commons licence and your intended use is not permitted by statutory regulation or exceeds the permitted use, you will need to obtain permission directly from the copyright holder. To view a copy of this licence, visit http://creativecommons.org/licenses/by-nc/4.0/.

\section{References}

1. Morgan DK, Domann DE, Collins GE, et al. History and evolution of field-based medical programs. Drug Inf J. 2000;34(4):1049-52.

2. Justia Trademarks. MSL - trademark details. 2020. https://trade marks.justia.com/731/76/msl-73176757.html Accessed 21 Dec 2020.

3. Accreditation Council for Medical Affairs (ACMA). 2018. https:// www.medicalaffairsspecialist.org/why-medical-affairs/ Accessed 21 Dec 2020.

4. Werling K, Carnell H, McCormick D. Focus on life science compliance: the evolution of Medical Affairs Departments. 2011. https ://www.mcguirewoods.com/news-resources/publications/healt 
h_care/focus-life-science-compliance-nov-2011.pdf. Accessed 21 Dec 2020.

5. Evens RP. Medical affairs and professional services. In: Evens RP, editor. Drug and biological development. Boston: Springer; 2007. https://doi.org/10.1007/978-0-387-69094-0_11.

6. Food and Drug Administration. FDA guidance document on responding to unsolicited requests for off-label information about prescription drugs and medical devices. $2011 \mathrm{https}: / / \mathrm{www} . \mathrm{fda}$. gov/regulatory-information/search-fda-guidance-documents/respo nding-unsolicited-requests-label-information-about-prescriptiondrugs-and-medical-devices? source=govdelivery. Accessed 21 Dec 2020.

7. L.E.K. Transforming the medical affairs function. Accessed 8 November 2018. https://www.lek.com/insights/ei/transformingmedical-affairs-function. Accessed 21 Dec 2020.

8. West Monroe Partners, rMark Bio. Using AI to align field engagements with strategy in medical affairs. 5 May 2020. https://www. biopharmadive.com/spons/using-ai-to-align-field-engagement s-with-strategy-in-medical-affairs/577043/. Accessed 21 Dec 2020.

9. Champagne D, Hung A, Leclerc O. The road to digital success in pharma. 1 August 2015. https://www.mckinsey.com/industries/ pharmaceuticals-and-medical-products/our-insights/the-road-todigital-success-in-pharma. Accessed 2 November 2020.

10. King S. Physician views: medical affairs will prove a key conduit for pharma's engagement with doctors as COVID-19 pandemic evolves, poll indicates. 17 April 2020. https://www.firstwordp harma.com/node/1716504. Accessed 2 Nov 2020.

11. World Health Organization Healthcare expenditure. https://data. worldbank.org/indicator/SH.XPD.CHEX.GD.ZS Accessed 21 Dec 2020.

12. Policy Advice. The state of healthcare industry (2020). https ://policyadvice.net/insurance/insights/healthcare-statistics/. Accessed 11 Oct 2020.
13. MIT Technology Review Insights. The AI effect: how artificial intelligence is making healthcare more human. 2019. https://mittr insights.s3.amazonaws.com/ai-effect.pdf. Accessed 21 Dec 2020.

14. Schuhmacher A, Gatto A, Hinder M, et al. The upside of being a digital pharma player. Drug Discov Today. 2020;25(9):1569-74.

15. Agrawal P. Artificial intelligence in drug discovery and development. J Pharmacovigil. 2018;6:2. https://doi.org/10.4172/2329$6887.1000 \mathrm{e} 173$.

16. Arsene C. Artificial Intelligence \& Pharma: what's next. 4 August 2020. https://www.digitalauthority.me/resources/artificial-intel ligence-pharma/ Accessed 21 Dec 2020.

17. SyTrue. Why unstructured data holds the key to intelligent healthcare systems. 31 March 2015. https://hitconsultant. net/2015/03/31/tapping-unstructured-data-healthcares-biggesthurdle-realized. Accessed 21 Dec 2020.

18. Woolley BG, Stanley KO. A novel human-computer collaboration: combining novelty search with interactive evolution. In: Proceedings of the 2014 Annual Conference on Genetic and Evolutionary Computation (GECCO '14). New York: Association for Computing Machinery; 2014; pp 233-240

19. Brown T, Mann B, Ryder N et al. Language Models are fewshot learners. 22 July 2020. https://arxiv.org/pdf/2005.14165.pdf Accessed 21 Dec 2020.

20. Armstrong HF. Medical affairs in the digital age. https://www. pharmalive.com/medical-affairs-in-the-digital-age/. Accessed 21 Dec 2020.

21. Evers M, Fleming E, Ghatak A, et al. Pharma Medical Affairs: 2020 and beyond. 2014. https://hsbgateway.com/documents/PR/ Pharma-Medical-Affairs-2020.pdf. Accessed 21 Dec 2020. 